\title{
Da produção à utilização de resultados de pesquisa na prática assistencial: uma experiência em consolidação
}

\author{
From the production to the use of research results in care practice: a consolidating experience
}

De la producción a la utilización de resultados de investigación en la práctica asistencial: una experiencia en consolidación

\author{
Emília Campos de Carvalho', Ana Maria Laus', Maria Helena Larcher Caliri', Luci Grupioni Rossi" \\ 'Universidade de São Paulo. Escola de Enfermagem de Ribeirão Preto. Ribeirão Preto, SP \\ "Universidade de São Paulo. Hospital de Clínicas da Faculdade de Medicina de Ribeirão Preto, Ribeirão Preto, SP
}

Submissão: $21 / 08 / 2009$

Aprovação: 20/06/2010

\section{RESUMO}

Embora desejável, nem sempre os profissionais estão preparados para consumir resultados de pesquisa. Este artigo busca apresentar o processo de desenvolvimento e implantação de inovações, baseado no modelo de Rogers, por meio de estratégias para preparar o enfermeiro para utilizar as melhores evidências para uma prática clínica de Qualidade. Trata-se de uma parceria entre uma instituição de ensino e uma de assistência hospitalar. A partir da identificação de problemas oriundos das áreas de atuação dos enfermeiros da instituição de saúde, foram desenvolvidos projetos de pesQuisas para a busca de evidências e testes de intervenções. Os resultados dessa experiência evidenciaram a viabilidade do uso do modelo apresentado como referencial metodológico, contribuindo para alcance das metas da instituição.

Descritores: Pesquisa em enfermagem; Educação em enfermagem; Hospitais de ensino.

\section{ABSTRACT}

Although it is desirable that professionals use research results, often they are not prepared for that. This article aims to present the process of development and establishment of innovations, based on Rogers' model, through strategies to train nurses to use the best evidences for a Quality clinical practice. It is a partnership between a educational institution and a hospital care institution. Following identification of problems from nurses' own practice in a health institution research proposals were developed to search for evidences and to test interventions. Results of this experience evidenced the viability of use of a model presented as a methodological framework what contributed to reach the institutions' goal.

Key words: Nursing research; Education, nursing; Hospitals, teaching

\section{RESUMEN}

Aún sea deseable, ni siempre los profesionales están preparados para utilizar resultados de investigación. Este artículo objetiva presentar el proceso de desarrollo y implementación de innovaciones, basado en el modelo de Rogers, por medio de estrategias para preparar el enfermero para utilizar las mejores evidencias para una práctica clínica de calidad. Se trata de una asociación entre una institución de enseñanza y una de atención hospitalaria. A partir de la identificación de problemas oriundos de las áreas de actuación de los enfermeros participantes del proyecto $(n=28)$, fue consensuado el desarrollo de 18 proyectos de investigación. Los resultados de este experimento demostró la viabilidad de utilizar el modelo que se presenta como un modelo metodológico, lo eue contribuye al logro de los objetivos de la institución.

Descriptores: Investigación en enfermería; Educación en enfermería; Hospitales escuela.

AUTOR CORRESPONDENTE Emília Campos de Carvalho. Avenida Bandeirantes, 3900. CEP 14040-902. Ribeirão Preto, SP.

E-mail: ecdcava@usp.br 


\section{INTRODUÇÃO}

A ação investigativa em Enfermagem é reconhecida como essencial no campo clínico, por possibilitar a aQuisição de conhecimentos, contribuindo para a avaliação da prática assistencial e crescimento profissional, dando maior visibilidade ao trabalho em Enfermagem ${ }^{(1)}$. Pode ser compreendida, ainda, como um estímulo para elevar a Qualidade científica, tecnológica e artística dos saberes profissionais de enfermagem ${ }^{(2)}$. Contudo, seu desenvolvimento ainda se dá com entraves tais como a não realização de investigações sobre os problemas na prestação de serviços de Qualidade e atenção de enfermagem; não utilização dos resultados de pesquisa para modificação das práticas em benefício da clientela; não capacitação dos enfermeiros de serviço em pesQuisa Que possam incorporar a prática da peseuisa no serviço e desconsiderar as responsabilidades com o ensino, fazendo com Que os resultados de pesquisa cheguem aos profissionais para além das publicações e eventos científicos ${ }^{(3)}$.

Embora se deseje Que esse profissional venha a se tornar consumidor de pesquisa, com vistas à evolução da prática de enfermagem e de saúde ${ }^{(4)}$, nem sempre, no seu processo de formação a pesquisa está incorporada como uma de suas atividades ${ }^{(3)}$.

Outro paradoxo é Que, apesar do aumento da produção do conhecimento em Enfermagem, não se observou significativos impactos na prática hospitalar, ainda centrada em desenvolvimento de tarefas. Diversos estudos apontam que os obstáculos para a utilização do conhecimento produzido estão voltados, prioritariamente, para a disseminação dos resultados desses estudos e àQueles relacionados à instituição e, secundariamente, aos fatores relacionados ao profissional ${ }^{(5)}$.

$\mathrm{Na}$ expectativa de se realizar uma prática assistencial fundamentada em conhecimento científico próprio, bem como buscando ultrapassar a etapa do ideal para o concreto, algumas estratégias já foram testadas. Estudo sobre a utilização da pesQuisa na prática clínica de enfermagem aponta Que no cenário internacional, desde a década de 1970, são desenvolvidos projetos para testar e consolidar o emprego de resultados de pesquisas na assistência ${ }^{(5)}$. Projetos dessa natureza ainda são incipientes em nosso meio.

No Brasil, identificou-se um estudo visando à aQuisição de competências em investigação científica, tendo como base a aprendizagem por descoberta, tutoria e estratégias do ensino humanista $^{(1)}$. Participaram do programa 86 enfermeiras, das Quais 70 concluíram seus projetos. Desses, 28 resultaram em trabalhos científicos, em sua maioria relatos de experiência, pesQuisas descritivas e exploratórias. Segundo as enfermeiras, o programa proporcionouIhes a aQuisição e aplicação de conhecimentos em pesQuisa.

Outra experiência, envolvendo as iniciativas de instalação de centros de pesquisa ${ }^{(6)}$, menciona Que foram executados treinamentos sobre metodologia de pesquisa, busca científica na internet além de cursos de "Desenvolvimento de competências e habilidades para operacionalização do processo de enfermagem na prática assistencial”, com a participação de diversos enfermeiros da instituição. Os resultados evidenciaram um engajamento gradativo nas atividades dos enfermeiros, com significativo interesse no conhecimento de metodologia de pesquisa.

Nota-se também Que as instituições hospitalares têm apoiado as iniciativas de criação de setores de Assessoria de Desenvolvimento e Pesquisa $^{(7)}$ ou de instalação de centros de apoio à pesquisa ${ }^{(8)}$ podendo o enfermeiro participar em diferentes papéis em tais centros, inclusive como pesquisador principal.

Nesse mesmo sentido, estudo ${ }^{(8)}$ aponta a experiência de um Centro em um Hospital Universitário, Que foi criado para apoiar as enfermeiras na realização de pesquisas. No período de 1999 a 2002, a produção científica dos enfermeiros compreendeu 129 projetos de pesquisa e 151 trabalhos científicos. Destes, 36 foram publicados em periódicos e seis premiados em eventos científicos. Obteve ainda relatos do desenvolvimento de estratégias e ações pela mobilização dos atores e da instituição com o despertar para a atividade investigativa, o criar na postura de realizadoras de pesquisa, com repercussões intra e extramuros à instituição, percebidas no desenvolvimento pessoal-profissional e na valorização do trabalho desenvolvido.

A integração de pesQuisadores de instituições acadêmicas com os profissionais das instituições da prática assistencial tem sido defendida desde a década de 70, com a finalidade de criar parcerias ao redor de problemas e metas comuns e contribuir para o avanço da produção e utilização do conhecimento assim como do "status" da enfermagem ${ }^{(5)}$. A proposta de integração surgiu a partir do pressuposto de Que a ligação entre membros de um mesmo grupo social, mas Que atuam em diferentes contextos como, por exemplo, o acadêmico e clínico pode fortalecer o grupo como um todo e levá-lo ao alcance de metas comuns frente a situações que demandam transformações, o Que não seria possível individualmente.

Os modelos de utilização do conhecimento, criados com esta ótica, têm como embasamento a teoria de difusão de inovações criada por Rogers ${ }^{(9)}$, um cientista social Que estudou o processo de decisão pela adoção, os seus componentes e as barreiras e estratégias para facilitar tal adoção. Na enfermagem, o enfoQue inicial desses modelos foi no processo de utilização de resultados de pesquisas que poderiam melhorar a assistência. Na década de 1990 esse enfoque se ampliou com a incorporação de outras evidências, como as diretrizes para a prática clínica embasadas na opinião de especialistas, em áreas onde os estudos científicos são escassos ou Quando não indicam a melhor forma de se proceder à frente ao problema.

A difusão de uma inovação em um sistema social não é espontânea, e ocorre em cinco estágios: (1) conhecimento, (2) persuasão, (3) decisão, (4) implementação e (5) confirmação; a rapidez da adoção depende de outros fatores como as características dos membros do sistema, da própria inovação assim como do tempo ${ }^{(9)}$. Este autor acrescenta Que o fato de uma inovação ser boa ou melhor do Que a prática tradicional não significa Que será adotada e, ainda, Que as barreiras para a sua adoção precisam ser identificadas de forma Que estratégias possam ser desenvolvidas e adotadas pelos responsáveis pela inovação.

$\mathrm{Na}$ atualidade, a integração de enfermeiros da instituição acadêmica e da instituição onde ocorre a prática clínica é apresentada como uma estratégia para a Qualificação dos profissionais em ambos os ambientes e para o desenvolvimento da competência para utilização das melhores evidências. O movimento mundial pela melhoria da Qualidade dos serviços de saúde das últimas décadas tem impulsionado esta integração com base na crença Que, Questões da Qualidade da assistência e segurança do 
paciente estão associadas aos custos e Que o uso das melhores evidências pelos profissionais com maior competência pode melhorar os resultados para a instituição e para os usuários dos serviços ${ }^{(10)}$.

A estrutura teórica dos modelos Que visam obter mudanças na prática e o uso das evidências (vistas como inovações) é complexa, pois considera o ambiente onde a prática ocorre, as características dos profissionais, a própria inovação e as diversas estratégias e ações Que vão permitir o avanço da inovação pelos diferentes estágios do modelo.

O propósito deste artigo é apresentar o processo de desenvolvimento e a implementação de um curso de capacitação para enfermeiros, baseado no modelo de Rogers e guiado pelas estratégias disponíveis na literatura Que visam a preparar o enfermeiro para utilizar as melhores evidências disponíveis para uma prática clínica de Qualidade.

A proposta ora relatada, uma parceria entre a Escola de Enfermagem de Ribeirão Preto da Universidade de São Paulo (EERPUSP) e a Divisão de Enfermagem do Hospital das Clínicas da Faculdade de Medicina de Ribeirão Preto da Universidade de São Paulo (HCFMRP-USP), considerou os seguintes pressupostos:

- Que o ensino da metodologia de pesquisa ainda é enfatizado apenas nos cursos superiores e muito pouco nas instituições empregatícias e Que uma das dificuldades para os enfermeiros realizarem pesquisas refere-se ao desconhecimento da metodologia e Que esse conhecimento não lhes foi transmitido na graduação ${ }^{(11-12)}$;

- Que em geral, o foco de ensino está centrado na elaboração e execução de um projeto de pesQuisa, sem a preocupação da continuidade e do engajamento dos alunos em projetos mais amplos de utilização ou em participação em núcleos de pesQuisa. E ainda, Que a pesQuisa, apoiada pela organização e desejada pela enfermeira, consubstancia-se como estratégia científico-pedagógica na formação permanente de enfermeiros assistenciais e agrega repercussões positivas para a assistência prestada;

- Que existe a necessidade de transformar o caráter teórico da disciplina metodologia da pesquisa para teórico-prático, por meio da implementação da pesQuisa de campo como estratégia de ensino ${ }^{(13)}$;

- Que ao acolher e desenvolver tema de pesquisas advindo de problemas ou dificuldades identificados pelos enfermeiros e promover a formação de uma rede de trabalho entre enfermeiros, educadores e pesQuisadores, amplia-se os horizontes, não só sobre os problemas do cotidiano, mas também sobre as possibilidades metodológicas para resolvê-los ${ }^{(1)}$;

- Que ao fortalecer o Quadro de enfermeiros com formação em pesquisa da própria instituição hospitalar, tais profissionais possam ser constituídos agentes de mudanças.

Destaca-se, ainda, Que a proposta apresentada vem ao encontro das diretrizes do Programa Nacional de Reorientação da Formação Profissional em Saúde - Pró-Saúde (MS/ME), Que estimula a realização de educação continuada dos profissionais da saúde, em especial sobre a peseuisa clínica para avaliação crítica do processo de atenção à saúde.

\section{A PROPOSTA E SEU DESENVOLVIMENTO}

Os docentes do Departamento de Enfermagem Geral e Especializada da EERP-USP ministram aulas para estudantes de graduação e pós-graduação assim como desenvolvem ensino clínico nas unidades de internação e ambulatórios do HCFMRP-USP. A diretora da Divisão de Enfermagem, os diretores dos Serviços de Enfermagem e os enfermeiros da instituição participam ativamente de várias atividades acadêmicas dos estudantes; assim, um projeto em parceria tem o potencial de beneficiar ambas as instituições, profissionais e estudantes.

O projeto, planejado de forma colaborativa entre as instituições, durante três meses, foi idealizado para ser desenvolvido em Quatro anos, visando a aplicabilidade das fases da Prática Baseada em Evidências $^{(14)}$.

Considerando Que o ambiente institucional onde a prática ocorre e as características dos profissionais podem facilitar ou dificultar a adoção de mudanças, a proposta foi configurada sob a forma de cursos planejados para Que os participantes pudessem vivenciar cada etapa do processo de construção da pesquisa clínica e de assessorias voltadas para a obtenção de melhor desempenho dos mesmos no desenvolvimento do projeto e a sua implantação, contribuindo para a melhoria da Qualidade da prática de enfermagem na instituição.

Foram previstos três cursos no projeto; cada um foi proposto isoladamente e exigiu cerca de três meses para sua aprovação nos órgãos acadêmicos. Seu planejamento contou com oito horas de atividades semanais, sendo Quatro horas para atividades teórico-práticas e Quatro horas para discussões, orientações e desenvol-vimento das tarefas de cada etapa, por onze meses. A negociação do número de encontros semanais foi uma estratégia para facilitar a liberação do participante do serviço e a realização da escala na Unidade.

Para o desenvolvimento dos cursos, colaboraram doze docentes da EERP; empregando diversas estratégias de ensino: aulas expositivas e dialogadas com discussões em grupos, estudos dirigido, busca de literatura e análises de publicações e elaboração de projeto pesquisa. Os critérios de avaliação exigiam a freqüência mínima de $85 \%$ nas atividades e nota mínima sete (7.0) na apresentação do projeto de pesQuisa.

Em 2007 foram propostos e realizados dois cursos para dois grupos de enfermeiros. A estratégia de ministrar o curso em horários distintos visou a favorecer a participação de o maior número de enfermeiros provenientes das mesmas unidades de internação, assim como obter maior envolvimento dos profissionais de uma mesma unidade de internação para implementar as mudanças identificadas na prática. Contudo, em 2008, em face de uma diretriz da instituição assistencial, o curso foi oferecido para enfermeiros, de diversas unidades da instituição, com atividades nos dois períodos do mesmo dia, aspecto Que favorecia maior aproveitamento das atividades práticas e de estudo. Tal decisão teve origem na sugestão dos participantes do primeiro grupo e foi reforçada pelas considerações de outros autores ${ }^{(1)}$ Que recomendam a orientação contínua e respeito às particularidades de cada enfermeiro.

A indicação dos enfermeiros foi feita pela diretoria da Divisão de Enfermagem do hospital, considerando as unidades em Que os docentes do curso desenvolvem suas atividades acadêmicas. Participaram desses cursos 39 enfermeiros assistenciais.

Foram estabelecidos os seguintes objetivos a serem alcançados pelos participantes:

- identificar as ações de enfermagem caracterizadas por problemas;

- identificar os aspectos éticos e legais nas situações estudadas; 
- identificar os aspectos éticos em pesQuisa clínica;

- identificar as etapas e métodos de uma pesquisa clínica e dos modelos de utilização da peşuisa

- identificar o papel e as funções dos enfermeiros em pesquisa clínica e nos modelos de utilização de pesquisas /evidências na prática de enfermagem;

- elaborar um projeto de pesquisa ou de utilização dos resultados da pesquisa, relacionado a um dos problemas da prática de enfermagem.

Após a explicação da dinâmica do curso, objetivos, estratégias e atividades, os enfermeiros deveriam optar por trabalharem sozinhos, em duplas ou em grupos de três, considerando o seu local de atuação ou tópico de estudo. Esta estratégia considerou o fato de Que durante todas as fases do curso os enfermeiros trabalhariam com Questões da prática Que identificassem como um problema e Que exigia exploração e avaliação, culminando ao final com uma proposta de intervenção.

A proposta global, com as atividades dos cursos e de acompanhamento (assessorias), foi planejada considerando as fases do modelo adotado ${ }^{(9)}$ e do modelo de lowa da Prática Baseada em Evidências para promover a Qualidade do cuidado $^{(14)}$, conforme descrito a seguir:

\section{ESTÁGIO DO CONHECIMENTO}

Esta fase compreendeu o desenvolvimento do curso, na Qual se buscou:

- Identificar um problema da prática clínica e caracterizá-lo destacando-se a reflexão de porQue o tópico era um problema e o Que já dispunha de conhecimento sobre o assunto, a partir das evidências oriundas de literatura, obtidas em eventos científicos, visitas a outros serviços ou com especialistas sobre o tema. Frente a um possível vazio ou aspecto negativo da assistência, os participantes eram levados a refletirem sobre a filosofia do cuidado adotado na instituição e Quais os reais problemas Que poderiam ser focalizados em um projeto científico visando a avaliação e transformação da realidade institucional do ponto de vista do enfermeiro e da instituição.

- Clarificar o problema e obter a descrição da forma como a assistência era feita considerando o problema com base na justificativa da importância para a instituição, para a enfermagem, paciente ou familiar.

- Realizar a pesquisa bibliográfica para seleção da literatura relevante e compreender a definição de palavras chaves, acesso e uso de bases de dados, leitura dos resumos, recuperação de artigos na versão $p d f, h t m l$ e em papel. Paralelamente, o conteúdo do programa abordou temas referentes aos métodos e técnicas de pesquisa bibliográfica, leitura e crítica de pesquisa, delineamentos de pesquisa Quantitativa, hierarQuia de evidências e sua relevância para a prática clínica.

Para estruturar a pergunta de investigação, tanto do projeto como do levantamento bibliográfico, bem como para a análise da literatura, utilizou-se os Quatro componentes do modelo $\mathrm{PICO}^{(10)}$, ou seja, a definição da População, Paciente ou Problema de interesse, da Intervenção (evidência nova), da intervenção Comparativa ou Cuidado tradicional e dos Resultados ou desfechos (Outcomes).

\section{Estágio da persuasão}

Nesta etapa o enfermeiro deveria construir uma opinião sobre o problema Que estava sendo investigado comparando o Que descreveu previamente sobre o seu contexto de trabalho e as recomendações disponíveis na literatura sobre as práticas mais adequadas e as evidências Que dão o embasamento. Para isso os participantes analisaram os componentes da publicação: leitura do material bibliográfico (às vezes precedida da tradução para o português), crítica, síntese e discussão entre duplas e posteriormente com o grupo e os coordenadores do curso. Ainda, deveriam definir se existiam evidências suficientes Que suportavam a mudança na instituição, assim como as possibilidades de implementação no contexto clínico, e se existiam situações ou pessoas que poderiam ser facilitadores, bem como as fragilidades ou barreiras Que precisariam ser enfrentadas.

Os enfermeiros sintetizaram as evidências disponíveis sobre o problema investigado e prepararam uma apresentação em PowerPoint, para os demais participantes do curso, coordenadores, chefia e diretoria da Divisão de Enfermagem da instituição, o Que além de possibilitar a experiência do preparo e treino para a exposição simulada na forma de evento cientifico, também objetivou obter a aprovação da Instituição e estímulo para Que projeto continuasse em suas fases subseQüentes.

Ao final da negociação a decisão poderia tanto levar a implementação inicial em unidade piloto ou a realização de pesQuisa. Esta estratégia favoreceu a discussão sobre a estrutura organizacional da instituição e como se deu o processo de decisão para implementação de inovações, assim como sobre os recursos necessários e os disponíveis para a adoção das mudanças.

Portanto, as atividades realizadas culminaram com elaboração de projetos de pesquisa, passíveis de serem realizados nos setores de trabalho dos participantes. Tais projetos deveriam conter todos os componentes de uma investigação incluindo o orçamento e os formulários para submissão às instancias regulatórias.

Foram elaborados 20 projetos, com temáticas Que se relacionavam aos principais problemas de cada setor, como por exemplo, sobre integridade tecidual; eventos adversos relacionados à sondas/ cateteres; programas educativos destinados a pacientes; satisfação do cliente; sistematização da assistência de enfermagem, dentre outros. Alguns projetos, por sua natureza inovadora, foram incluídos no planejamento estratégico institucional para a melhoria da Qualidade; tal planejamento está apoiado pelas Comissões de Controle de Infecção Hospitalar, Gerenciamento de Riscos, Comitê de Segurança do paciente e de Educação Permanente.

Merece destaque o fato de Que durante o ano de 2008, ao mesmo tempo em Que transcorria o terceiro curso, foram agendadas reuniões bimensais para acompanhamento e ajustes dos projetos para os alunos ingressantes em 2007. Nesta época a instituição não estava ainda concedendo uma carga horária mensal ao profissional destinada exclusivamente para o desenvolvimento da pesquisa. Tal estratégia não favoreceu a execução das atividades de pesquisas programadas. Em 2008, para os egressos dos três cursos, foram estabelecidos horários fixos correspondendo a um turno de trabalho, mensalmente, para orientações e acompanhamento dos trabalhos, considerados como atividade de educação permanente.

Ao se avaliar os aspectos facilitadores e bloqueadores para o 
desenvolvimento dessa proposta, destacamos a concordância entre tais achados e as premissas inicialmente propostas pela literatura ${ }^{(1,11-}$ ${ }^{13)}$, as Quais nortearam a realização desta experiência. Destacamos ainda o caráter positivo do despertar dos enfermeiros para a realização de pesquisas baseadas em suas vivências, nas áreas de atuação, com o propósito de identificar e mensurar indicadores de desempenho de enfermagem em suas unidades de trabalho, uma das metas da instituição. Por fim, sugerimos a adoção da tutoria nos horários de estudo, aspecto Que favorece a elaboração do projeto e desenvolvimento da pesQuisa propriamente dita.

\section{Estágio de decisão}

A maioria dos responsáveis pelos projetos de pesQuisa decidiu, juntamente com os diretores de enfermagem e coordenadores da proposta, pelo desenvolvimento dos mesmos; os demais participantes se encontram ainda na fase de decisão.

\section{Estágio de implementação}

O modelo teórico adotado ${ }^{(9)}$ prevê duas etapas para este estágio: a utilização de evidências disponíveis na literatura ou o desenvolvimento de estudos Que gerem as evidências a serem implantadas. No caso ora descrito os participantes do curso optaram pela segunda alternativa e deverão, após sua conclusão, procederem à incorporação dos resultados destas pesquisas na prática.

As atividades desta etapa visam implementar as mudanças na prática e se iniciam pela criação de um plano e protocolo Que será testado em uma unidade piloto. A avaliação dos resultados da mudança deve considerar Quais aspectos serão mensurados antes e depois da implementação e Quais instrumentos serão utilizados para a coleta e análise dos dados, assim como os desfechos serão divulgados e utilizados pelo enfermeiro responsável pelo projeto e pela instituição ${ }^{(14)}$.

A presente proposta encontra-se nesta fase e conta com o acompanhamento, pelos coordenadores, dos trâmites aos órgãos competentes e instâncias regulatórias para a realização de alguns dos projetos, bem como da coleta de dados para as demais pesQuisas. A seguir ocorrerão a análise dos dados e a apresentação dos resultados e sua posterior divulgação, tanto sob a forma de artigo como de exposição oral ou escrita aos demais enfermeiros da instituição assistencial, além dos aspectos supra citados.

\section{Estágio da confirmação}

Os resultados obtidos na etapa anterior, se positivos, poderão fortalecer a decisão já adotada de criar as mudanças e levar a disseminação da inovação na instituição, com a implementação em suas outras unidades. Se forem avaliados como negativos ou ainda se o impacto produzido não justificar os custos ou o tempo gasto, o processo de mudança será interrompido. Tais passos deverão estar em concordância com os demais programas institucionais, avaliados continuamente, por meio dos indicadores de Qualidade ou outro programa adotado pela instituição. Estas duas fases estão previstas para realização até 2010 .

\section{Contribuições iniciais da proposta}

A educação tem sido considerada um instrumento de mudanças e transformações no âmbito da saúde. A acumulação de conhecimento, traduzido em tecnologias e indicadores da Qualidade dos processos de trabalho, tem influenciado a organização do trabalho, exigindo Que os trabalhadores adeuiram novas habilidades ${ }^{(15)}$.

Neste sentido, instituições de saúde Que tem se inserido em programas de avaliação de Qualidade, necessariamente são impelidas a promover uma revisão nos seus processos de trabalho e na prestação de serviços. O gerenciamento, neste ambiente, tem como pressuposto uma gestão participativa, uma co-responsabilização pela melhoria da assistência, Que significa a introdução de mecanismos sistemáticos de avaliação.

Há Que se considerar o aspecto integrador, social e político, Que o processo de produção e divulgação de uma inovação pode ter com vistas à cooperação entre seus usuários e o bem estar social ${ }^{(16)}$.

O HCFMRP-USP, credenciado pelo Ministério da Saúde, desde 2005, como hospital de ensino, aderiu voluntariamente ao plano de metas e avaliação permanente de seus resultados, no âmbito de desempenho da sua gestão hospitalar. De acordo com o Ministério da Saúde ${ }^{(17)}$, o desenvolvimento de programas de garantia de Qualidade é uma necessidade em termos não só de eficiência, mas se constitui numa obrigação do ponto de vista ético e moral.

A instituição estabeleceu, portanto, uma política de gerenciamento de Qualidade e implementou um sistema de avaliação e monitoramento por meio de indicadores de desempenho nos diferentes departamentos Que compõe sua estrutura organizacional, dentre eles a Divisão de Enfermagem, visando a promover a utilização sistemática destas informações como subsídio para os processos de melhoria contínua da assistência hospitalar.

As recomendações emanadas dos programas de avaliação para as instituições de saúde, no Que se referem a uma política de segurança do paciente, estabelecem adoção de sistemas de informação com dados e indicadores, com evidências de ciclos de melhoria da assistência, incluindo atividades e processos voltados para a capacitação e Qualificação da força de trabalho, entre outros ${ }^{(17)}$.

A importância do despertar dos enfermeiros para a realização de pesouisas baseadas em suas vivências nas áreas de atuação com o propósito de identificar e mensurar indicadores de desempenho de suas unidades de trabalho, portanto, configura-se em uma das metas da instituição, em seu Projeto de Qualidade. Frente aos indicadores de Qualidade e recomendações de itens a serem tratados no Programa institucional de Qualidade, verificamos uma coerência com os projetos propostos pelos enfermeiros, já descritos.

Ainda, em consonância ao modelo adotado, alguns resultados preliminares já foram observados, em face de algumas estratégias estabelecidas pela Divisão de Enfermagem:

- preparo de enfermeiros para atuarem no Centro de Pesquisa Clínica, recentemente instalado na instituição;

- o estímulo para que maior número de enfermeiros participe do estudo dos indicadores de assistência de enfermagem, e busca na literatura de evidências Que sustentem ou modifieuem a prática assistencial;

- estímulo para Que enfermeiros participem da replicação de estudos disponíveis na literatura ${ }^{(2)}$ e a avaliação, para o cenário escolhido, de evidências de outros estudos, para subsidiar a prática.

Espera-se Que em curto prazo a instituição consolide uma comissão de apoio aos projetos de investigação de enfermagem, e Que, à semelhança de experiência de outros serviços ${ }^{(2)}$, seja possível constituir uma linha de pesquisa, oriunda do próprio grupo de enfermeiras com temas de interesse comum. 
Um dos maiores desafios para as instituições de saúde é criar cada vez mais envolvimento e comprometimento dos profissionais. Para tanto, ao oferecer oportunidades de crescimento e desenvolvimento, espera-se Que se estabeleça uma responsabilização para com os objetivos da organização Que é o de prestar uma assistência Qualificada. Nesta direção esta experiência indica Que as enfermeiras ao investirem no desenvolvimento de competências essenciais, identificadas pelo conhecimento técnico-assistencial acompanhado da prática gerencial, respondem às necessidades de um profissional Que utiliza o conhecimento para dar resolutividade aos problemas e propor melhorias na instituição ${ }^{(18)}$.

\section{CONSIDERAÇÕES FINAIS}

A proposta relatada considerou os pressupostos de Que o conhecimento e implantação de uma inovação passam pelas fases de persuasão (para testar/utilizar a inovação), decisão pelos diferentes níveis organizacionais se aceita ou rejeita a mudança ou inovação; implementação da inovação (plano para o seu uso) e confirmação ou avaliação da utilização da inovação (relatos de seus resultados e decisão de sua continuidade ou não).

Esta experiência amplia as reflexões sobre a capacitação dos enfermeiros no seu processo de trabalho por uma instituição parceira e a sua vinculação com os resultados pretendidos no Que tange à Qualificação da assistência prestada. Há de se considerar os aspectos avaliados como centrais para o desenvolvimento de programas desta natureza como: o caráter inovador para a instituição de ensino, o envolvimento e investimento da instituição assistencial na equipe de enfermagem e a ampliação dos campos de atuação em unidades hospitalares ou na indústria farmacêutica com pesQuisa clínica.

\section{REFERÊNCIAS}

I. Dyniewicz A M, Gutiérrez MGR. Metodologia da pesquisa para enfermeiras de um hospital universitário. Rev Latino-am Enfermagem 2005; 13(3): 354-63.

2. Trentini M, Paim L. Assistência e pesQuisa em enfermagem: uma abordagem convergente assistencial. Texto Contexto Enferm $2001 ; 10(1): 11-31$.

3. Cabral IE, Tyrrel MAR. Pesquisa em enfermagem nas Américas. Rev Bras Enferm 2010; 63(1): 104-10.

4. Servo MLS, Oliveira MAN. A pesquisa e o enfermeiro com Qualidade formal e Qualidade política: caminho para a consolidação da enfermagem como ciência Sitientibus. Rev Univ Estadual Feira Santana 2005; 33:1 1-21.

5. Caliri MHL. A utilização da pesquisa na pratica clínica de enfermagem. Limites e possibilidades [tese de livre docência]. Ribeirão Preto: Escola de Enfermagem de Ribeirão Preto, Universidade de São Paulo; 2002.

6. Zapata MRCG, Bezerra ALQ, Coelho MA, Azevedo AS, Oliveira MW, Valente NMLM. Grupo de pesquisa em enfermagem: relato de experiência. In: Anais do $57^{\circ}$ Congresso Brasileiro de Enfermagem; 2005 nov 5-7; Goiânia (GO), Brasil. Goiânia: ABEn; 2005.

7. Lima IA, Alvarenga D, Bettarello LEL. A Enfermagem desencadeando o processo de mudança organizacional. RAS 2001; 3(10): 17-20.

8. Guariente MHDM, Zago MMF. Produção científica de enfermeiros assistenciais com apoio de assessoria em pesQuisa. Rev Latino-am Enfermagem 2006; 14 (3): 1-7.

9. Rogers EM. Diffusion of innovations. $5^{\text {th }}$ ed. New York: Free Press; 2003.

10. Melnyk BM, Fineout-Overholt E. Evidence-based practice in nursing \& healthcare. A guide to best practice. Philadelphia: Lippincott Williams \& Wilkins; 2005.

11. Cassiani SHB. Buscando significado para o trabalho: o aperfeiçoamento profissional sob a perspectiva da enfermeira [tese]. Ribeirão Preto: Escola de Enfermagem de Ribeirão Preto, Universidade de São Paulo; 1994.

12. Lopes CM. Pesquisar para assistir. Rev Esc Enfermagem USP. 1992; 26: 105- 18.

13. Guimarães BMA, Morosini MC, Pinto AMB, Sturn A. Subsídios para um programa de treinamento de pesquisadores universitários. Rev Gaúcha Enferm 1985; 6(1):5 5-61 .

14. Titler MG. Uso da peseuisa na prática. In: LoBiondo-Wood, G; Haber I, organizadores. Pesquisa em Enfermagem: métodos, avaliação crítica e utilização. $4^{a}$ ed. Rio de Janeiro: Guanabara Koogan; 2001. p. 268-87.

15. Ricaldoni CAC, Sena RR. Educação permanente: uma ferramenta para pensar e agir no trabalho de enfermagem. Rev Latino-am Enfermagem 2006; I 4(6): 837-42.

16. Martini JG. O papel social da peseuisa em Enfermagem. Editorial. Rev Bras Enferm 2009; 62(3): 340.

17. Ministério da Saúde (BR). Secretaria de Políticas da Saúde. Departamento de Avaliação de Políticas de Saúde. Manual Brasileiro de Acreditação Hospitalar. 2aed. Brasília: Ministério da Saúde; 1999.

18. Ruthes RM, Cunha ICKO. Competências do enfermeiro na gestão do conhecimento e capital intelectual. Rev Bras Enferm 2009; 62(6): 901-5. 